\title{
Study on the Heat Transfer Characteristics Performed in the Infrared Thermography Detection of Welded Structure
}

\author{
Zhiping Liu, Xingle Liu*, Lei Jiang, Ge Lu and Huilong Liu
}

School of Logistics Engineering, Wuhan University of Technology, Wuhan, Hubei, 430063, P.R. China

\begin{abstract}
During the weld defect detection, the heat transfer characteristics are closely related to the material properties of welded structure. Based on the electromagnetic induction infrared thermography technology, the heat transfer Characteristics of the welded material are studied with the changing temperature. By using the finite element analysis software COMSOL, the eddy current density and temperature distributions and the law of thermal diffusion were analyzed which provide a reference for the study of heat transfer characteristics of weld defects. The internal temperature dynamic changes of the weld with surface crack or near-surface crack were also discussed. The appropriate time to observe and the key defect location on the steel obtained from the heat conduction process can be applied to the development of heat transfer characteristic analysis for steel weld and weld defects detection.
\end{abstract}

Keywords: Electromagnetic induction, heat transfer characteristics, simulation analysis, the material properties, welded structure.

\section{INTRODUCTION}

As one of the most important connection method of metal, welded structures have the advantages of simple, easy processing, better connectivity, stiffness, connection with good performance and great flexibility, etc. They are widely used in various industries fields, such as bridges, ships, automobiles, high-pressure containers. However, due to the existence of welding defects or concentrated stress, when it is under cyclic loading, the fatigue crack initiation in the weld is extended to a critical size and it is the main cause of a fatigue accident [1].

In the course of the detection of welded structure, with the advantages of non-contact, swift detection, high degree of sensitivity, the infrared thermography testing technology is developed as an emerging type of non-destructive testing method and has been widespread concerned in recent years $[2,3]$. Compared to the external optical excitation, internal ultrasonic vibration and other infrared thermal imaging incentive method, electromagnetic excitation becomes the first choice of the detection method because of its high heating efficiency, excellent stability and reproducibility. Electromagnetic induction infrared thermography is a kind of detecting method that defects are identified according to the abnormal heat distribution. The heat transfer characteristics and weld defect detection are closely related to the material properties of welded structure. Based on the electromagnetic induction infrared thermography, by the use of finite element analysis software COMSOL4.3b, the temperature and eddy current distributions in the heat transfer process of the conductor are analyzed in this paper. The material properties performed in the heat transferring process under 3 cases: a) weld with free-defect; b) surface crack; c) near-surface crack, are respectively obtained, which provide a further theoretical guidance on the weld defect detection and the study of heat transfer characteristics.

\section{PRINCIPLE AND MATHEMATICAL MODEL}

\subsection{Principle of the Research on Heat Transfer Characteristics}

The principle on detecting steel weld defect is based on electromagnetic induction infrared thermgraphy method. According to the law of electromagnetic induction, alternating current is input into the induction coil which is placed above the detection area of the conductor. Eddy current will be generated on the conductor surface of the specimen. If there is any defect, the eddy current will bypass the defect which results in a change of eddy current density in partial area. According to Joule's law, the heat generated by the conductor material will exhibit a nonuniform distribution. The infrared imager is used to observe and record the surface temperature distribution of the specimen. The relationship between the thermal image and the defect is analyzed so as to realize the detection of defects in the conductor, as shown in Fig. (1) [4, 5].

\subsection{Mathematical Model}

Electromagnetic and thermal field analysis are coupled as the mathematical model in accordance with the testing process. to find the thermal characteristics changes of steel welded structure in the temperature field over time. Analyses of heat transfer properties in the steel weld are studied below.

\subsubsection{Eddy Current Field}

According to the law of electromagnetic induction, an alternating magnetic field will be produced near a conductor 
with an alternating current. The enclosed eddy current is generated on the conductor due to the magnetic field. Therefore, the conductor is defined as turbulent zone while the electromagnetic induction coil and air are non-turbulent zone.

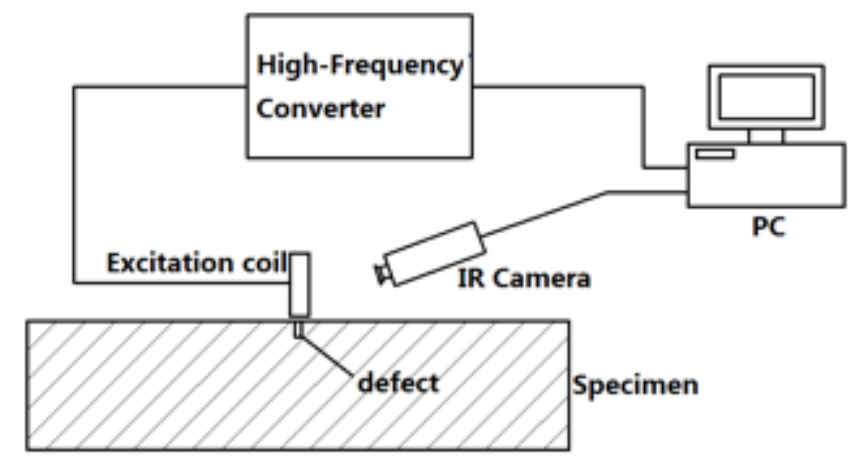

Fig. (1). The principle diagram of Eddy current thermography.

According to Maxwell's equation and Eigen equation, the magnetic and electric fields can be expressed as:

$$
\begin{aligned}
& B=\nabla \times A \\
& E=-\frac{\partial A}{\partial t}-\nabla \phi
\end{aligned}
$$

where,

$B$---the magnetic flux density, $T$;

$A$---electric displacement vector, $\mathrm{C} / \mathrm{m}^{2}$;

$E$---the electric field strength, $\mathrm{V} / \mathrm{m}$;

$\varphi$---the scalar potential.

By vector operations, control equation of eddy current field can be obtained:

$\nabla \times\left(\frac{1}{\mu} \nabla \times A\right)+\sigma \frac{\partial A}{\partial t}=\bar{J}$

where,

$\sigma$---the electrical conductivity, $\mathrm{S} / \mathrm{m}$;

$\mu$---the relative magnetic permeability;

$\bar{J}$---the external current density, $\mathrm{A} / \mathrm{m}^{2}$.

According to Faraday's law of electromagnetic induction, when the depth of plane conductor is infinite, eddy current density exponentially with the increased distance from the conductor surface. Penetration depth $\delta$ is used to indicate the attenuation speed of the delta amount of the electromagnetic field inside the conductor. Skin effect is strongly linked to the heat penetration depth $\delta$. Actually, in the induction heating duration, it is considered that energy is produced in the surface layer of the metal first and the interior part is heated by heat transfer. In general, the current penetration

depth $\delta$ is calculated as formula below:

$\delta=5030 \sqrt{\rho / f \mu}$

where, $\rho$---the resistivity, $\Omega \cdot \mathrm{cm}$;

$f$---the power frequency, $\mathrm{Hz}$.

Before the ferromagnetic material is completely lost the magnetic, eddy current penetration depth is referred to "cold vortex penetration depth." If the temperature of the material increased, then resistivity $\rho$ the penetration depth $\delta$ increases; when the temperature rises sufficiently to allow the material loses its magnetism, then $\mu$ will decay fast, and in turn, eddy current penetration depth will increase dramatically. Thus, when the material magnetic is lost completely, the eddy current penetration depth is called "thermal penetration depth of the eddy currents."

\subsubsection{Penetration Heating}

After switching on the current in the induction coil, at the moment that the temperature of the tested material starts to be significantly higher, eddy current penetration depth of the material is consistent with the cold vortex penetration depth in Eq. (4). The strength of eddy current will be increased and a sharp rise will be appeared in the strength of the material surface temperature when it is closer to the surface of the tested material. Part of the surface temperature of the material maybe exceed to a magnetic loss temperature. In this case, the material has two heating layers, one layer is magnetic lost, the other isn't. The magnetic permeability $\mu$ will be decreased fast because of the loss of magnetism. Eddy current strength will be quickly decayed in the magnetic loss layer and the highest eddy current intensity will be appeared at the critical areas of two layers. Due to the changes in the distribution of eddy current intensity, the material surface temperature rising rate is not as fast as the critical layer's mentioned above. Thus, the magnetic loss layer will be continuously be deeper. The material will be continuously heated layer by layer and not stopped until the penetration depth $\delta$.

\subsubsection{Thermal Conduction}

After eddy current penetration thickness of hightemperature layer which is lost of magnetic exceeds the thermal penetration depth $\delta$ of eddy current, heated continuously, the circulated heat of the material is basically from the layer of thickness $\delta$ from the surface. However, the closer the loss magnetism layer is to the surface, the higher energy the material and the eddy current intensity become. At the same time, due to the thermal conduction effect, the penetration thickness of the heating layer will get thicker with the increasing heating time. When the increased thickness of the material heated layer is far more than the material's eddy thermal penetration depth at the current frequency, the heating mode of the heating layer is thermal conduction.

\subsubsection{Temperature Field}

Obtained from the eddy current field, Joule heat generated from the induced current is used as an internal heat source to heat the specimen. The temperature distribution inside the conductor is determined by the eddy current distribution and heat transfer. As a result of the skin effect, the eddy current in the conductor, a transient signal, is unevenly distributed. The eddy current induction heating process with a nonuniform heat source is belonged to a unsteady heat transfer 
process and the established equation is a time-varying transient equations. temperature field control equation can be set up by the law of conservation of energy and Fourier law.

The temperature field and the eddy current field are coupled by using Joule's law. The heating power (internal heat density or intensity) generated by eddy current is represented by Q as Eq. (5) [6-8]:

$Q=\frac{1}{\sigma}\left|J_{e}\right|^{2}=\frac{1}{\sigma}|\sigma E|^{2}$

$\sigma=\frac{\sigma_{0}}{1+\alpha\left(T_{2}-T_{0}\right)}$

where,

$\sigma_{0}$---the conductivity at reference temperature $T_{0}, \mathrm{~S} / \mathrm{m}$;

$\alpha$---the temperature coefficient of resistance;

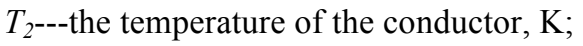

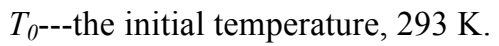

According to Fourier's law, the thermal conductivity of power (heat flow rate) per unit area $q$ is proportional to the temperature drop:

$q=-k \nabla T$

The temperature of the specimen at each point is unstable and the change per unit volume in the internal energy is:

$\rho C_{p} \frac{\partial T}{\partial t}$

Three-dimensional heat transfer equation can be obtained according to the induction heating energy conservation law and shown as below:

$\rho C_{p} \frac{\partial T}{\partial t}-\nabla \cdot k \nabla T=Q$

where,

$\rho$---the material density, $\mathrm{kg} / \mathrm{m}^{3}$;

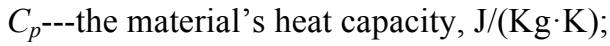

$k$---the thermal conductivity, $\mathrm{W} /\left(\mathrm{m}^{2} \cdot{ }^{\circ} \mathrm{C}\right)$;

Its differential form can be shown as:

$\frac{\partial}{\partial x}\left(k \frac{\partial T}{\partial x}\right)+\frac{\partial}{\partial y}\left(k \frac{\partial T}{\partial y}\right)+\frac{\partial}{\partial z}\left(k \frac{\partial T}{\partial z}\right)+Q-\rho C_{p} \frac{\partial T}{\partial t}=0$

Since the short heating time, typically several hundred milliseconds, properties of the conductor are constants.

Heat convective of the conductor is mainly considered during the cooling process:

$Q^{\prime}=h_{1} \cdot\left(T_{2}-T_{1}\right)$

where,

$Q^{\prime}$----the amount of heat, $\mathrm{J}$;

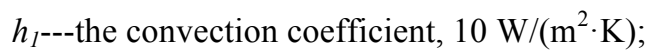

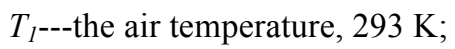

$T_{2^{---}}$the temperature conductor, $\mathrm{K}$.

\section{SIMULATION AND NUMERICAL ANALYSIS}

Finite element analysis software COMSOL4.3 is used to build steel weld models with free-crack, surface crack and near-surface crack respectively. Heat transfer characteristics in the process of heating and cooling duration are analyzed on weld crack detection.

As shown in Fig. (2), a simulation model without defect is established. The dimension of the specimen is: $400 \mathrm{~mm}$ $(L) \times 200 \mathrm{~mm}(W) \times 10 \mathrm{~mm}(\delta)$. In the middle, a V-shaped butt weld is provided as Fig. (3). The length of the weld is $200 \mathrm{~mm}(l)$, the reinforcement is $2 \mathrm{~mm} \mathrm{(h),} \mathrm{bevel} \mathrm{angle} \mathrm{is}$ $60^{\circ}(\alpha)$ and the groove depth is $8 \mathrm{~mm}(H)$. The weld material is supposed to be same as the base material.

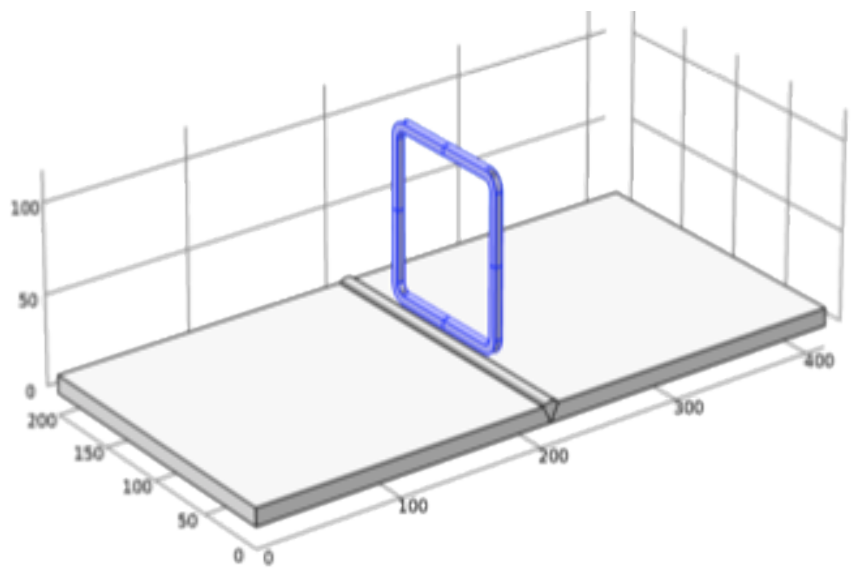

Fig. (2). The simulation model without defect.

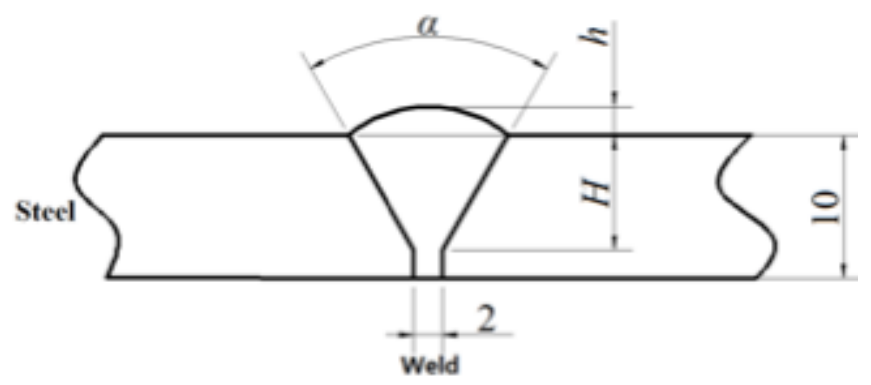

Fig. (3). The diagram of V-shaped weld.

Steel weld model with surface crack is shown in Fig. (4). The dimension of the crack is $20 \mathrm{~mm} \times 0.5 \mathrm{~mm} \times 0.5 \mathrm{~mm}$. It is located under the coil and paralleled to the weld.

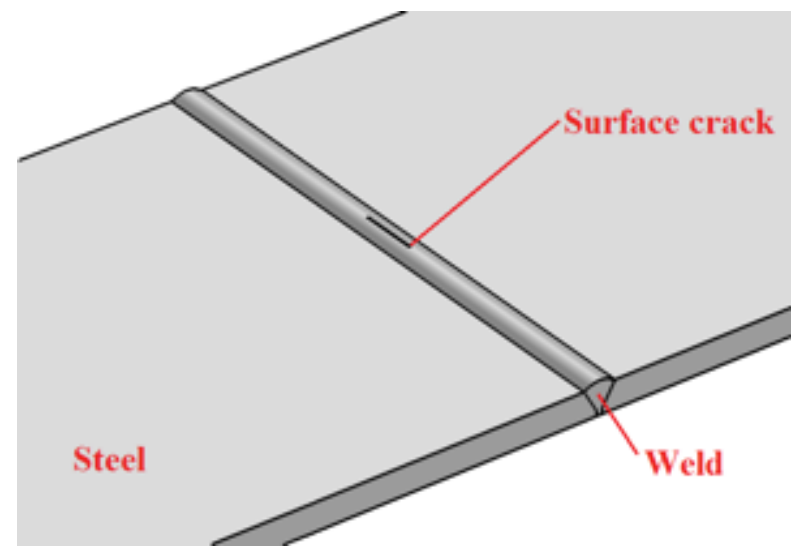

Fig. (4). The diagram of surface crack. 
The diagram of sectional schematic of weld with nearsurface crack is shown in Fig. (5). The dimension of the crack is $20 \mathrm{~mm} \times 0.5 \mathrm{~mm} \times 0.5 \mathrm{~mm}$. It is located below the coil, at a distance of $0.5 \mathrm{~mm}$ from the weld surface.

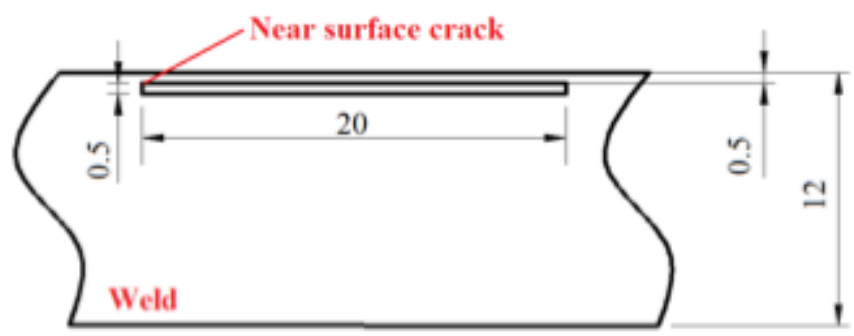

Fig. (5). The diagram of sectional schematic of weld with nearsurface crack.

The side-length of the square coil is $100 \mathrm{~mm}$, crosssectional dimension is $5 \mathrm{~mm} \times 5 \mathrm{~mm}$ and the liftoff height is set as $8 \mathrm{~mm}$. Input current through the cross-section of induction coil, the frequency of the excitation pulsed current is $250 \mathrm{kHz}$ while the amplitude is $350 \mathrm{~A}$. The heating duration is set as $200 \mathrm{~ms}$ and the cooling one is $300 \mathrm{~ms}$. The boundary conditions that conductor area is set as continuous and air as magnetic insulation.

Material parameters used in the simulation are shown in Table 1.

In the heating process of the electromagnetic excitation, the thermal physical properties of the metal material--45\#steel (i.e., relative permeability, electrical resistivity, thermal conductivity, specific heat capacity, etc.) are changed with the changing temperature are shown in Table 2 .

\subsection{Analysis of Heat Transfer in the Defect-Free Weld}

After heated by eddy current induction for $200 \mathrm{~ms}$, eddy current and temperature distributions of steel weld surface are in Fig. (6).

Eddy current distribution shows that in the weld, eddy current flows in the longitudinal direction of the weld. The maximum temperature is appeared just below the coil and diffusely decreased. according to the temperature distribution. The central point of the steel weld surface is selected as a characteristic point and analysis of the temperature dynamic process of the heating and cooling duration is shown in Fig. (7):

(a)

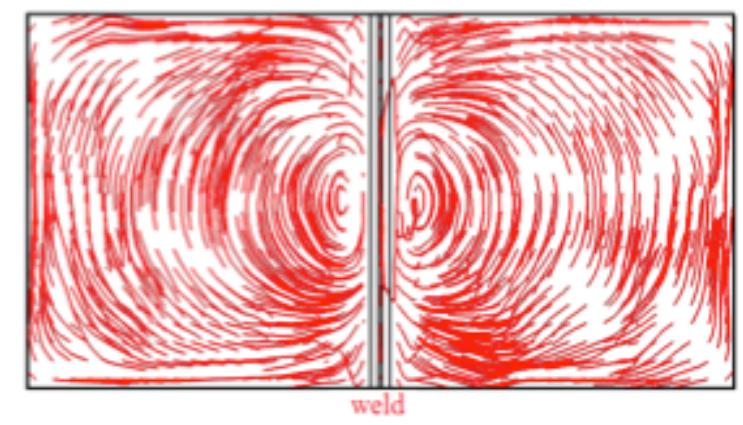

(b)

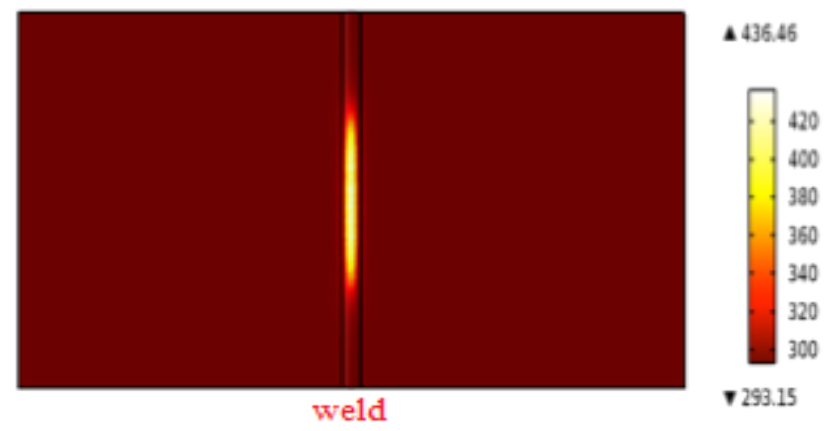

Fig. (6). (a) Eddy current (b) Temperature distribution of weld surface without defect [K].

Three directions are included in the analyses of heat transfer dynamics of weld without defects: a) surface in the width direction of the weld; b) surface in the longitudinal direction; c) along the depth direction of the weld, are shown in Fig. (8).

Table 1. Thermo-physical properties of air.

\begin{tabular}{|c|c|c|c|c|}
\hline Parameters & Electrical Conductivity $\boldsymbol{\sigma}(\mathbf{S} / \mathbf{m})$ & Relative Permeability $\boldsymbol{\mu}$ & Heat Capacity $\boldsymbol{C}_{\mathrm{p}} \mathbf{J} /(\mathbf{k g} \cdot \mathbf{K})$ & Thermal Coefficient $\boldsymbol{k} \mathbf{W} /\left(\mathbf{M} \cdot{ }^{\circ} \mathbf{C}\right)$ \\
\hline \hline air & 0 & 1 & 1.005 & 0.0257 \\
\hline
\end{tabular}

Table 2. The temperature characteristics of steel plate thermal parameters.

\begin{tabular}{|c|c|c|c|c|}
\hline Parameters $\mathbf{T}\left({ }^{\circ} \mathrm{C}\right)$ & Relative Permeability & Resistivity $\times 10^{-6}(\mathrm{~m} \cdot \Omega)$ & Electrical Conductivity $\mathrm{W} /(\mathbf{m} \cdot \mathbf{K})$ & Heat Capacity $J /(\mathbf{k g} \cdot \mathbf{K})$ \\
\hline 20 & 200 & 0.198 & 47.68 & 472 \\
\hline 100 & 195 & 0.254 & 43.53 & 480 \\
\hline 200 & 186.6 & 0.339 & 40.44 & 498 \\
\hline 300 & 178.1 & 0.435 & 38.13 & 524 \\
\hline 400 & 167.1 & 0.541 & 36.02 & 560 \\
\hline 500 & 154.9 & 0.656 & 34.16 & 615 \\
\hline 600 & 137.8 & 0.79 & 31.98 & 700 \\
\hline
\end{tabular}




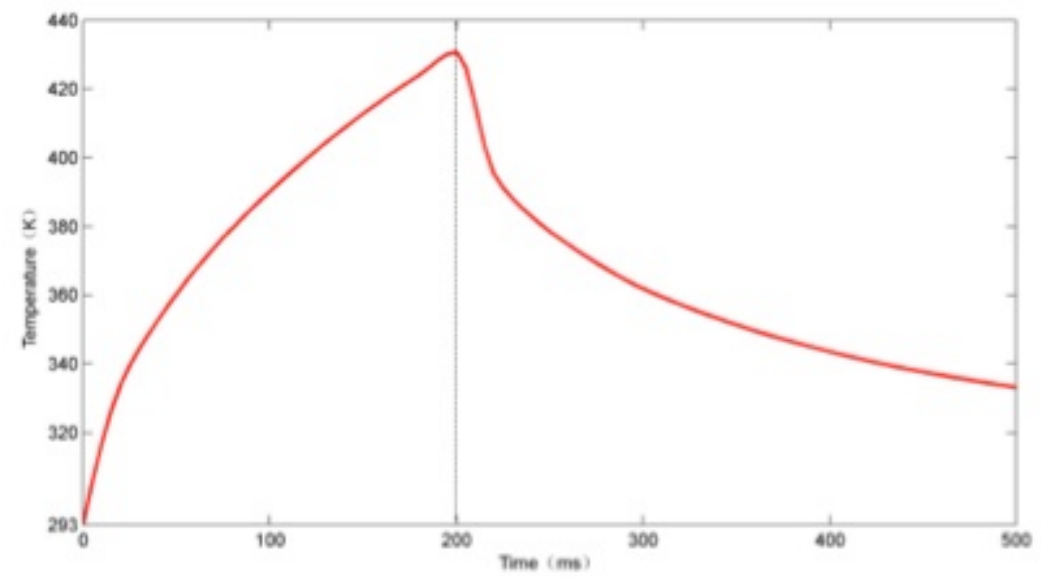

Fig. (7). Temperature dynamic process of the characteristic point on the weld surface.

(a)

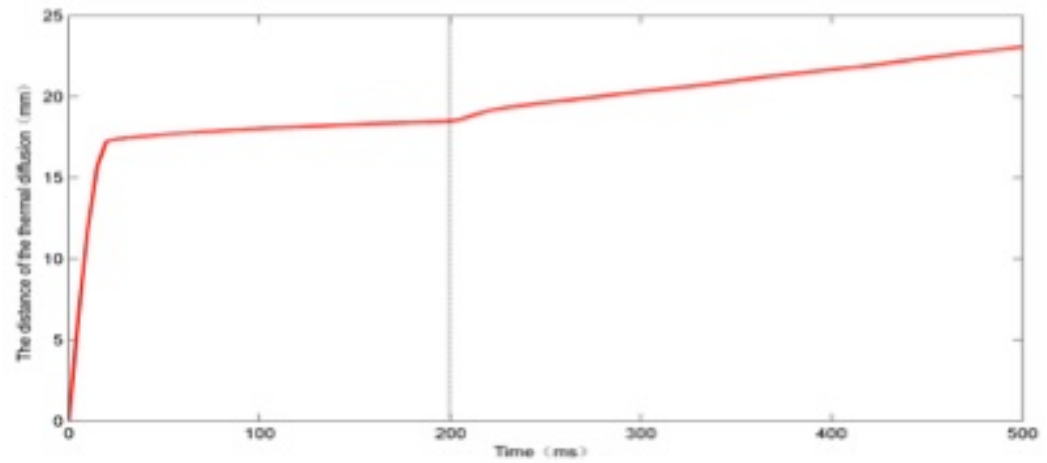

(b)

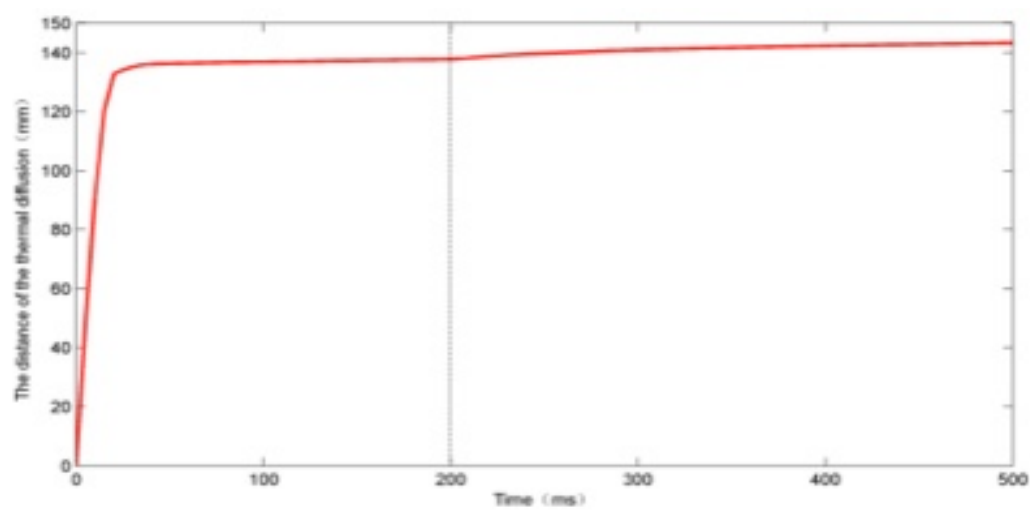

(c)

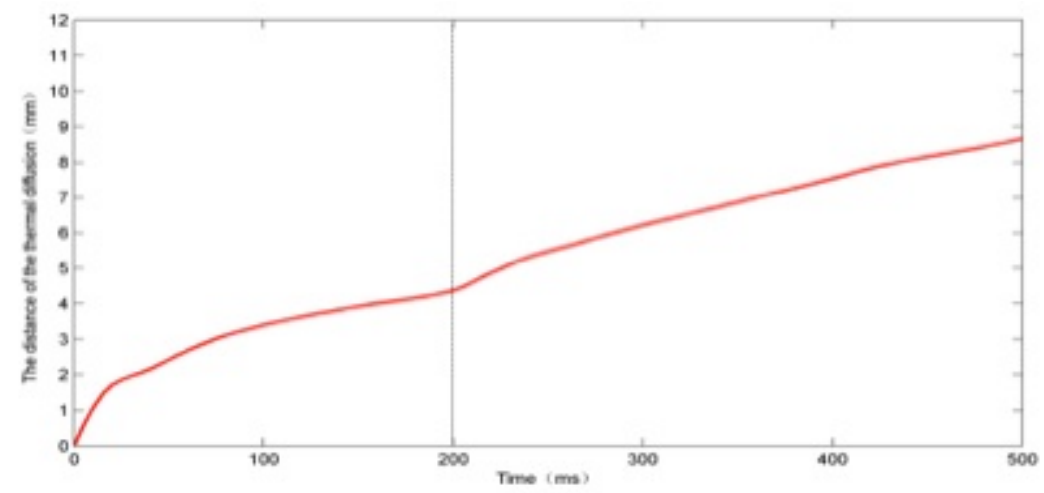

Fig. (8). Surface transfer in the (a) width; (b) longitudinal; (c) depth direction of the weld. 
From the view of Fig. $(\mathbf{8 a}, \mathbf{b})$, in the heating and cooling duration, the temperature diffusion law of the weld surface is concluded that the heat diffusion rate along the length direction of the weld is larger than the rate in the width direction. Namely, the temperature gradient along the longitudinal direction of the coil is larger than that in its width direction. Obtained from Fig. (8c), in the heating and cooling process, rates of heat diffusion are same in the depth direction of the weld.

\subsection{Heat Transfer Analysis of Surface Crack}

The dynamic temperature distribution around the weld surface crack is shown in Fig. (9). A section perpendicular to the surface of the steel and along the length of the crack is selected as object and the temperature distributions at 200 $\mathrm{ms}$ (heating) and $300 \mathrm{~ms}$ (cooling) are studied as Fig. (10). From the analysis of the heat transfer process, it can be obtained that the temperatures at both ends of the crack are higher than the one in other defective areas. The appropriate observation time in the thermography window is $100 \mathrm{~ms}-240$ ms.

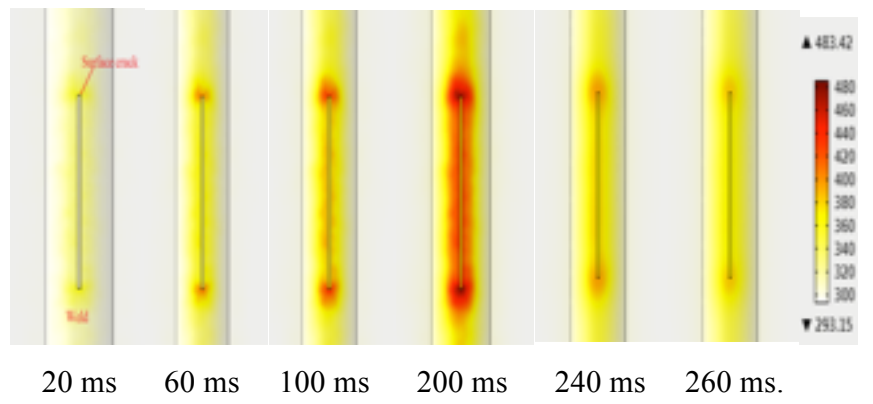

Fig. (9). The temperature nephogram of surface crack [K].

The edge line along the length of the weld crack is selected to be the characteristic object. Its eddy current distribution at $200 \mathrm{~ms}$ is researched. As shown in Fig. (11), along the characteristic line, there are two peaks of eddy current density. The distance between two peaks approximately equals to the length of the crack. Combined with the temperature distributions of surface and the crosssection of the weld at $200 \mathrm{~ms}$, it can be concluded that the increasing density of the eddy current results in the temperature rise at both ends of the crack on the surface.
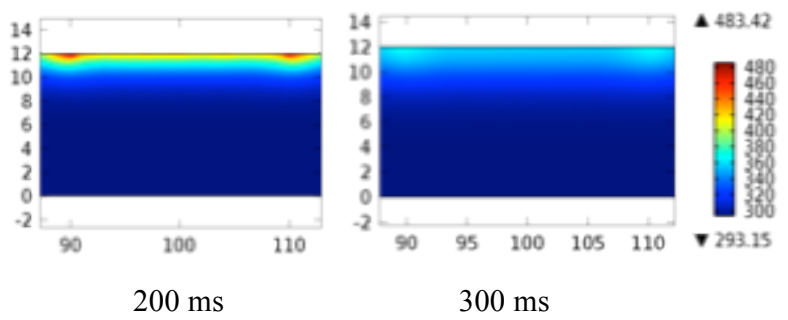

Fig. (10). The temperature distribution on specific cross-section of weld with surface crack [K].

Three characteristic points near the weld surface cracks are selected for analysis, where characteristic point 1 is at crack tip; point 2 is at the midpoint of the crack tips; point 3 is disposed in the non-defective area for comparison. As shown in Fig. (12), the rate of temperature changes of point 1 and 2 are significantly higher than of point 3. Namely, selecting feature points as the objects of study is more conducive to defect recognition in the study of surface cracks in steel welds.

\subsection{Heat Transfer Analysis of Near-Surface Crack}

The dynamic temperature distribution around the weld near-surface crack is shown in Fig. (13). A section perpendicular to the surface of the steel and along the length of the crack is selected as object and the temperature distributions at $200 \mathrm{~ms}$ (heating) and $300 \mathrm{~ms}$ (cooling) are studied as Fig. (14). By analyzing the process of heat conduction, the temperature of the area where is directly above the weld near-surface crack is higher than the temperature of other regions of the weld. The appropriate observation time in the thermography window is $100 \mathrm{~ms}-240 \mathrm{~ms}$.

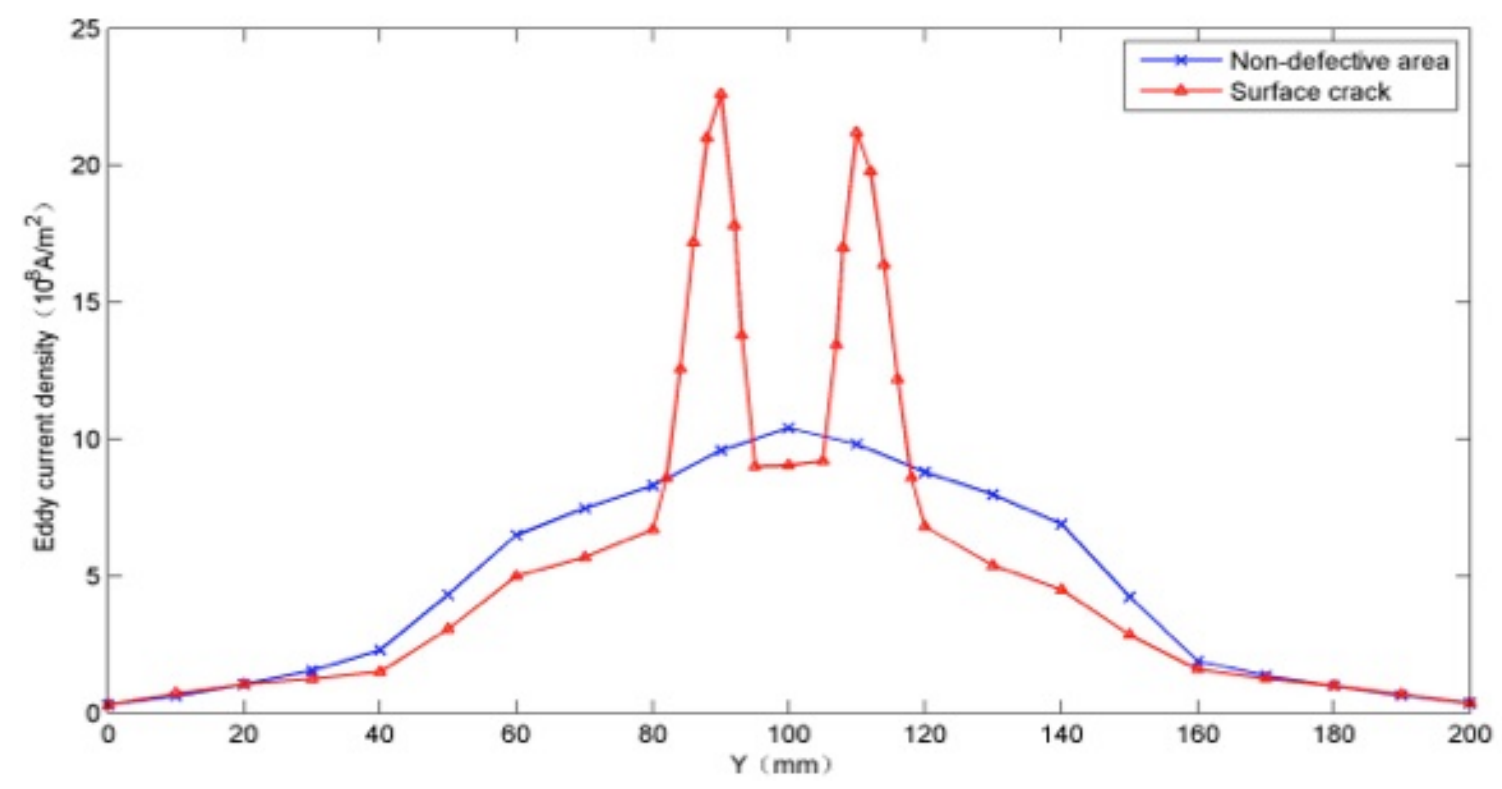

Fig. (11). Eddy current distribution of the surface characteristic line. 


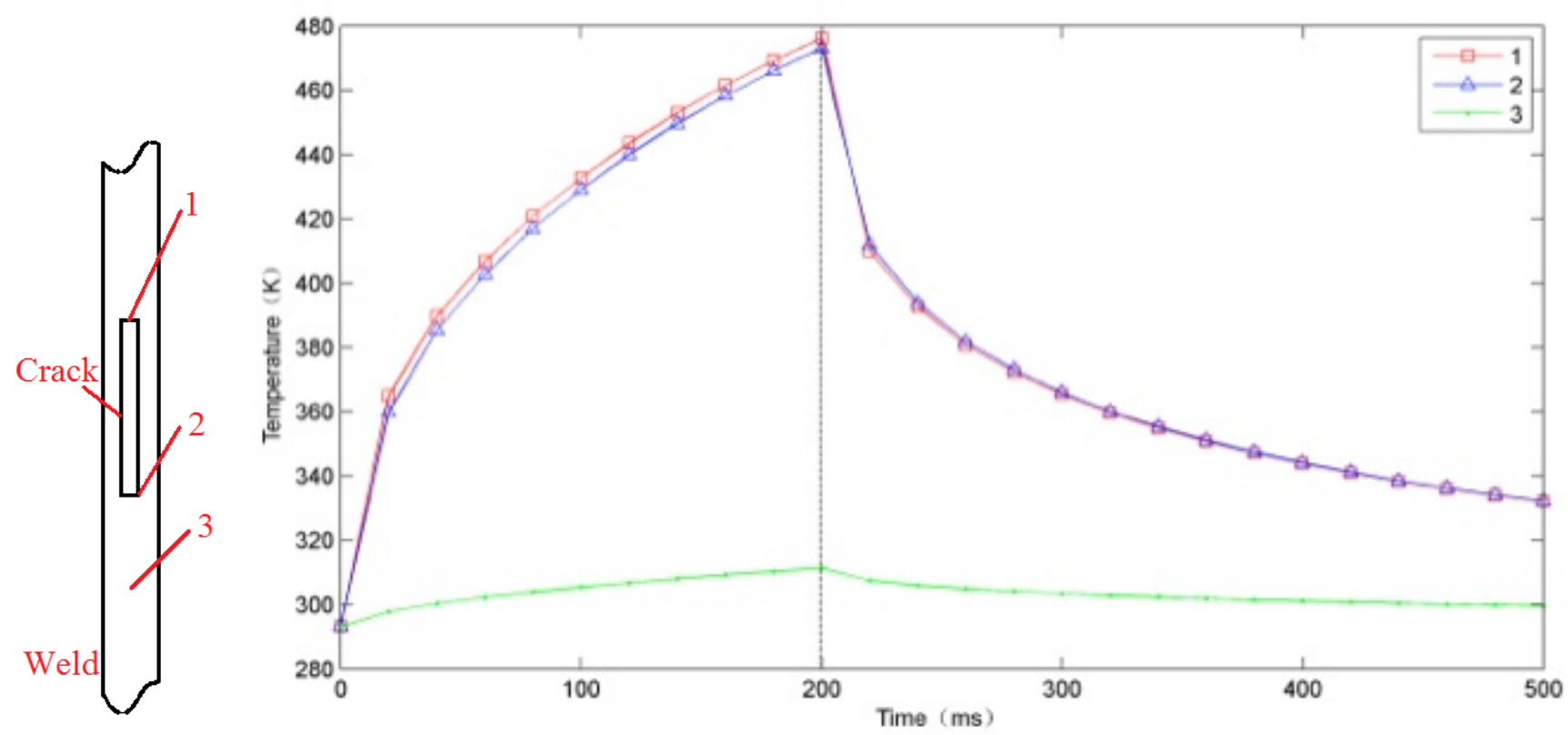

Fig. (12). Temperature changes at the characteristic points.

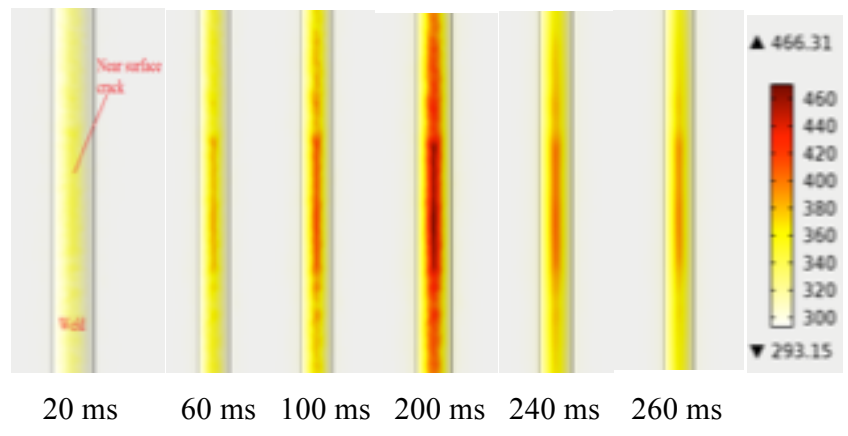

Fig. (13). The temperature nephogram of near-surface crack [K].
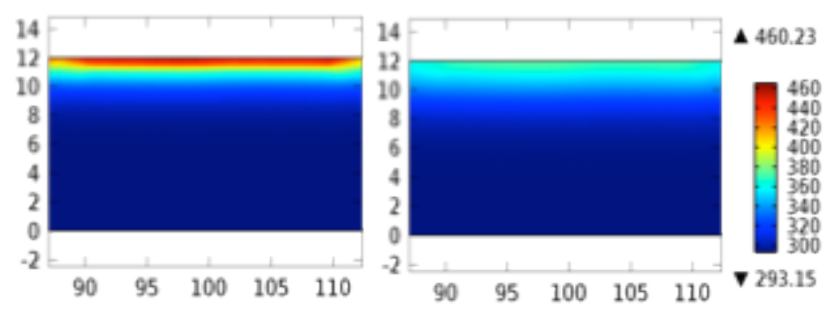

$200 \mathrm{~ms} 300 \mathrm{~ms}$

Fig. (14). The temperature distribution on specific cross-section of weld with near surface crack [K].

As shown in Fig. (15), in the direction of the weld, the projection of the weld edge line on the surface is chosen to be a characteristic line of the study. The eddy current distribution at $200 \mathrm{~ms}$ is shown in Fig. (16). Along the longitudinal direction of the weld, two peaks of eddy current density are appeared which the distance between them approximately equals to the length of the crack. Combined with the temperature distributions of the projection on the surface and the cross-section of the weld at $200 \mathrm{~ms}$, the increasing density of the eddy current results in the temperature rise at both ends of the crack on the surface.

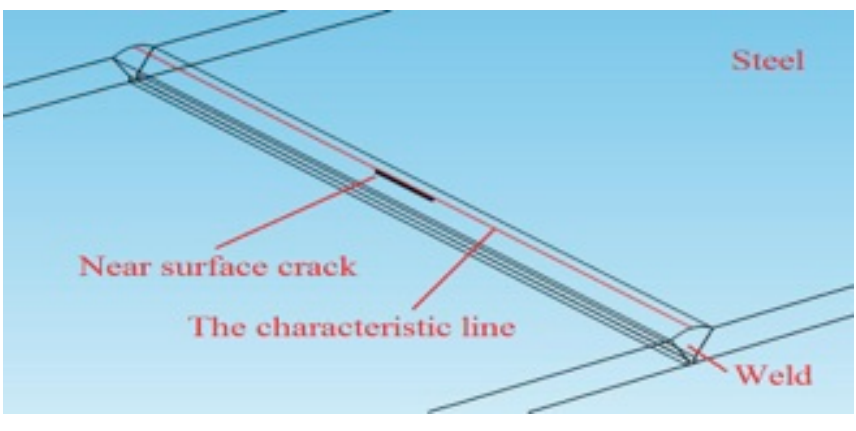

Fig. (15). Characteristic line of near surface crack.

To further detect the abnormal temperature distribution with steel weld defects, at $200 \mathrm{~ms}$ which is one of the best observation time, surface around the crack on the weld is selected as research object. Comparisons among the threedimensional temperature distributions of steel weld with defect-free, surface crack and near-surface crack are made as Fig. (17).

As shown above, at $200 \mathrm{~ms}$, due to the presence of surface or near surface crack, the temperature of the defective area is significantly higher than the temperature of the corresponding region with the absence of defects. Compared to the near-surface crack, temperatures at both ends of the surface cracks are higher than the surroundings. Thus, in the actual detection, where there is an abnormally high temperature range, characteristic points or lines can be selected as the object. Objective laws of heat transferring and the used method are detected to be further verified.

\section{CONCLUSION}

By building the eddy current heated mathematical model and using the finite element method, the simulation analyses on heat conduction dynamic process of weld with defect-free 


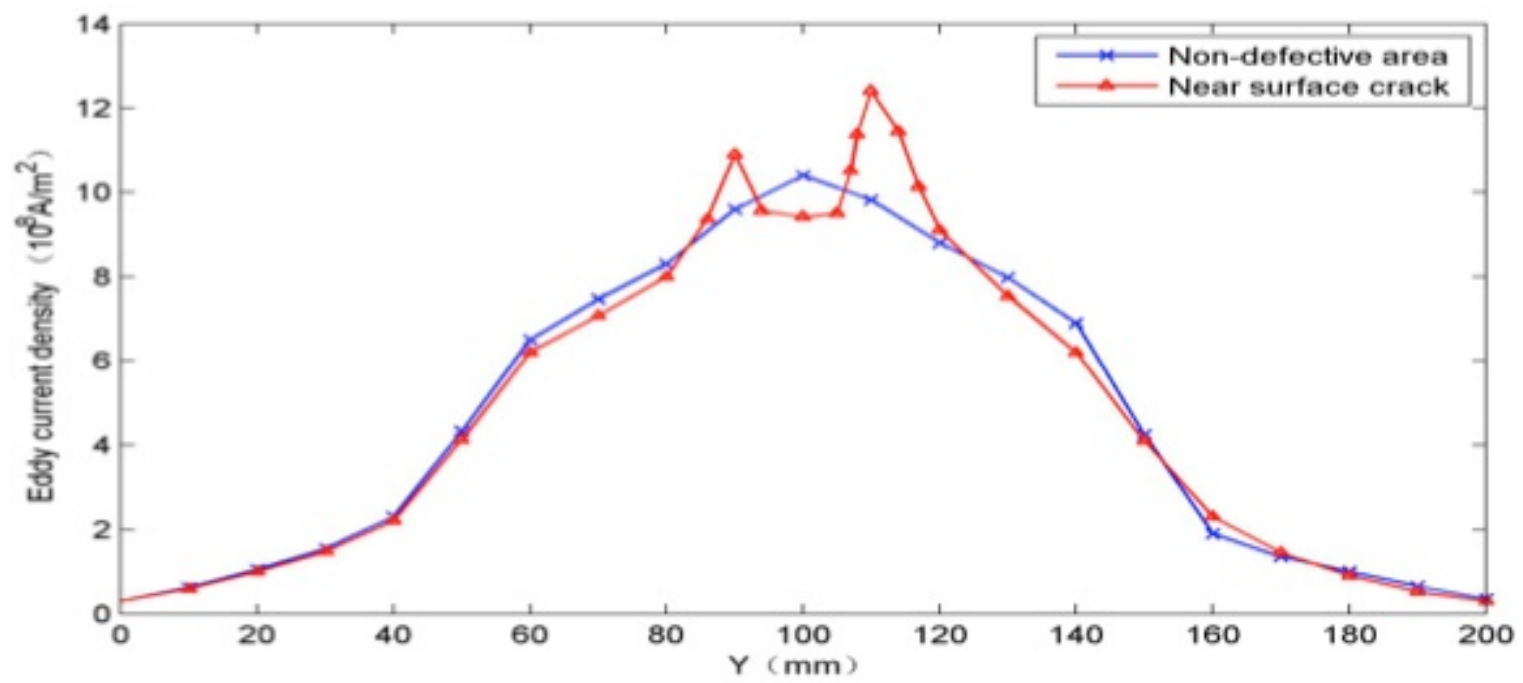

Fig. (16). The eddy distribution of the characteristic line.

crack, surface crack and near-surface crack are respectively made when material properties changed with the temperature.

(a) no crack

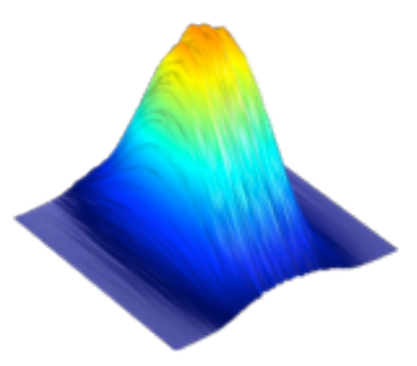

(b) surface crack

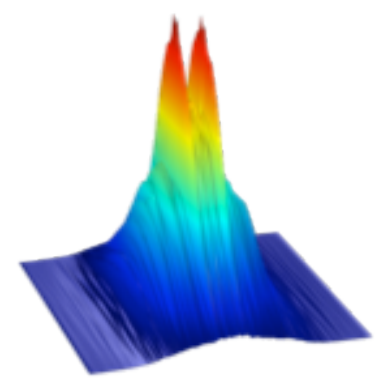

(c) near surface crack

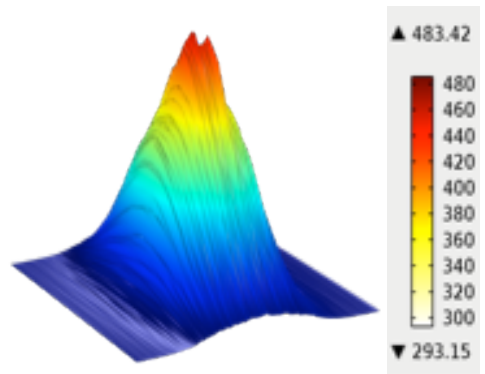

Fig. (17). 3-D Temperature distribution of 3 cases [K].
Conclusions are drawn as following: 1) When the defectfree weld is studied, eddy current, temperature distribution of weld surface and heat conduction dynamic process along three directions, the weld width, the longitudinal and the weld depth direction, are obtained, which provide a reference for the study of heat transfer characteristics of weld defects; 2)Due to the presence of steel weld surface crack and nearsurface crack, analyses of the dynamic changes of heat conduction are compared to pointed out the appropriate observation time in the thermography window can be applied to the development of heat transfer characteristic analysis for steel weld and weld defects detection; 3) The temperature distributions of non-defective weld steel, surface crack and near-surface crack are taken for comparison. Abnormal distribution would be generated if weld cracks exist which theoretical guidance is provided in the further studies of the heat transfer characteristics and the heating infrared detection of the welded materials. The weld micro-structure changes of different materials in the process of heating will be studied in the following research.

\section{CONFLICT OF INTEREST}

The authors confirm that this article content has no conflict of interest.

\section{ACKNOWLEDGEMENTS}

Financially supported by self-determined and innovative research funds of WUT.

This work was also supported by the appied fundamental research of the Ministry of Transport of the People's Republic of China (2014329811320).

\section{REFERENCES}

[1] P. Broberg, "Surface crack detection in welds using thermography", NDT \& E International, vol. 57, pp. 69-70, July 2013.

[2] Y.Y. Hung, Y.S. Chen, S.P. Ng, L. Liu, Y.H. Huang, B.L. Luk, R.W.L. Ip, C.M.L. Wu, and P.S. Chung, "Review and comparison 
of shearography and active thermography for nondestructive evaluation“, Materials Science and Engineering, vol. 64, pp. 73112, May 2009.

[3] C. Meola, "A new approach for estimation of defects detection with infrared thermography", Material letters, vol. 61, pp. 747-750, February 2007.

[4] J. Wilson, G.Y. Tian, I.Z. Abidin, S. Yang, and D. Almond, "Modelling and evaluation of eddy current stimulated thermography", Nondestructive Testing and Evaluation, vol. 25, pp. 205-218, 2010 .

[5] Z. Zeng, L. Wang, Y. Wang, and H. Zhang, "Numerical and experimental investigation on temperature distribution of thediscontinuous welding," Computational Materials Science, vol. 44, pp. 1155-1156, 2009 .

[6] H.S. Carslaw, and J.C. Jaeger, Conduction of Heat in Solids, Oxford University Press: Oxford 1986.

[7] I. Z. Abidin, G. Y. Tian, J. Wilson, S. Yang, D. Almond, "Quantitative evaluation of angular defects by pulsed eddy current thermography", NDT \& E International, vol. 43, pp. 538-539, 2010 .

[8] M. Noethen, J. Yi, and N. Meyendorf, "Simulation of the surface crack detection using inductive heated thermography", Nondestructive Testing and Evaluation, vol. 27, pp. 142-145, 2012.

(C) Liu et al.; Licensee Bentham Open.

This is an open access article licensed under the terms of the Creative Commons Attribution Non-Commercial License (http://creativecommons.org/licenses/by-nc/3.0/) which permits unrestricted, non-commercial use, distribution and reproduction in any medium, provided the work is properly cited. 